\title{
An Efficient Approach for Solving Fractional Dynamics of a Predator-Prey System
}

\author{
Hegagi Mohamed Ali ${ }^{1} \&$ Ismail Gad Ameen ${ }^{2}$ \\ ${ }^{1}$ Department of Mathematics, Faculty of Science, Aswan University, Aswan 81528, Egypt. \\ ${ }^{2}$ Department of Mathematics, Faculty of Science, South Valley University, Qena 83523, Egypt. \\ Correspondence: Hegagi Mohamed Ali, Department of Mathematics, Faculty of Science, Aswan University, \\ Aswan 81528, Egypt. E-mail: hegagi_math@aswu.edu.eg \& Ismail Gad Ameen, Department of Mathematics, \\ Faculty of Science, South Valley University, Qena 83523, Egypt. E-mail: ismailgad@svu.edu.eg
}

Received: July 30, 2019

Accepted: October 23, 2019

Online Published: October 24, 2019

doi:10.5539/mas.v13n11p116

URL: https://doi.org/10.5539/mas.v13n11p116

\begin{abstract}
In this work, we execute a generally new analytical technique, the modified generalized Mittag-Leffler function method (MGMLFM) for solving nonlinear partial differential equations containing fractional derivative emerging in predator-prey biological population dynamics system. This dynamics system are given by a set of fractional differential equations in the Caputo sense. A new solution is constructed in a power series. The stability of equilibrium points is studied. Moreover, numerical solutions for different cases are given and the methodology is displayed. We conducted a comparing between the results obtained by our method with the results obtained by other methods to illustrate the reliability and effectiveness of our main results.
\end{abstract}

Keywords: fractional partial nonlinear system, Mittag-Leffler function, predator-prey model, stability

\section{Introduction}

In recent years, the fractional differential equations have been used to describe many phenomena in various fields in engineering, physics, chemistry, and other sciences, such as electromagnetic waves, boundary layer effects in ducts, viscoelastic mechanics, the diffusion of biological populations, and so on Metzler\& Klafter (2000), Hilfe (2000), Oldham \& Spanier (1974), Podlubny (1999), Samko et al. (1993). However, most of these differential equations containing fractional derivative are very difficult to exactly solve, so numerical and approximation methods must be applied. There are many powerful methods have been used to approximate linear and nonlinear fractional differential equations. These methods involve homotopy perturbation method (HPM) He (2005), Ganji\& Sadighi (2007), Adomain decomposition method (ADM) Adomian (1988), variational iteration method (VIM) Safari et al. (2009), He (2011), generalized Mittag-Leffler function method (GMLFM) for ordinary fractional differential equations Arafa et al. (2013a,b) and so on.

The Mittag-Leffler function has gained great importance because of its participation in solving many applications of fractional differential equations. So, the method of the GMLFM proved its efficiency and its lightness in solving the ordinary fractional differential equations Arafa et al. (2013a,b). Moreover, the Mittag-Leffler function undetermined coefficient method is contributed to the solution of fractional homogeneous partial differential equations Liu et al. (2017).

It should be noted that the results obtained in this research are completely different from the results published in Liu et al. (2017) where we are using a different approach. As the method used in the mentioned paper solve only one partial fractional differential equation and the nonlinear part in this equation is always equal to zero, and this is not the case in our method presented in this article.

The Lotka-Volterra equations, also is called predator-prey equations, are a pair of first order differential equations utilized to characterize the dynamics of interactions of two species. Several authors studied first order systems and analyzed the dynamical behavior of these systems (see e.g. Gourley \& Britton (1996), Petrovskii et al. (2005)). The fractional-order predator- prey have been considered Ahmed et al. (2007), were the authors used fractional-order derivative instead of first-order time derivative. Recently, HPM in Kadem \& Baleanu (2011) is applied to solve the coupled fractional Lotka-Volterra equations. Liu \& Xin (2011) obtained a new analytical technique for solving nonlinear fractional partial differential equations arising in predator-prey biological 
population dynamics system. Finally, H. El-Saka (2014) considered the fractional-order predator-prey model and studied the stability of the equilibrium points (for more details see Ahmed et al. (2004), Petras (2011) and reference therein).

In our work we apply the MGMLFM to derive analytic approximate solutions of the following fractional system of partial differential equations Liu \& Xin (2011):

$$
\left\{\begin{array}{l}
{ }^{C} D_{t}^{\alpha} U(x, y, t)=a U-b U V+U_{x x}+U_{y y}, \\
{ }^{C} D_{t}^{\alpha} V(x, y, t)=b U V-c V+V_{x x}+V_{y y},
\end{array}\right.
$$

where ${ }^{C} D_{t}^{\alpha}$ is the Caputo fractional derivative of the functions $U$ and $V$ with respect to time $(t>0)$, for arbitrary order $\alpha$ (where $0<\alpha \leq 1$ ), $x, y \in \mathrm{R}$, the positive constants $a, b, c$ denotes the natural birth rate, the competitive rate and the natural death rate, respectively, and $U(x, y, t)$ and $V(x, y, t)$ represents the prey population and predator population density, respectively. The main reason for considering the predator-prey equations of fractional order $\alpha$ (where $\alpha \rightarrow 1$ ) is to lessen the error that may emerge from disregarded parameters or rearrangements in the classical predator-prey model. In this view, the utilization of $\alpha<1$ has just the effect of transforming the classical model in Liu \& Xin (2011) into a model with memory (1). Generally, fractional order partial differential equations are generalizations of integer order partial differential equations. Also, the importance of non-locality property for the fractional derivative make the solutions of the fractional system (1) at any time affected by all previous responses. Hence, again the fractional model (1) possess memory (where memory effects are important) (see e.g Du et al. (2013), Moaddy et al. (2012)).

This paper is organized as follows. In the next Section, we present some basic concepts of fractional calculus specifically pertinent to this work for understanding our main results are presented in this article. In Section 3, we formulate the MGMLFM for solving a general system of partial differential equations containing fractional derivative. Section 4 is devoted to study the equilibrium points and the stability analysis of the fractional system (1). We apply the MGMLFM to obtain analytic approximate solutions of the system (1) with two different cases of initial conditions in Section 5. Moreover, we show some numerical simulations of our model and compare the results with different methods to prove the efficacy of our approach. In Section 6 some conclusions are summarized.

\section{Some Preliminaries and Concepts}

Here, we provide some definitions and preliminaries which are related to the results of this paper (see e.g. Podlubny (1999), Samko et al. (1993)).

Definition 1. The Riemann-Liouville fractional integral of order $\alpha>0$ of a function $f(t)$, can be defined as

$$
\begin{aligned}
I_{t}^{\alpha} f(t) & =\frac{1}{\Gamma(\alpha)} \int_{t_{0}}^{t}(t-\xi)^{\alpha-1} f(\xi) d \xi, \quad t_{0} \geq 0, t>t_{0}, \\
I_{t}^{0} f(t) & =f(t),
\end{aligned}
$$

where $\Gamma(\cdot)$ is the Euler gamma function.

Definition 2. The Caputo partial fractional derivative of a function $g(x, t)$, of order $\alpha$ is defined as

$$
{ }^{C} D_{t}^{\alpha} g(x, t)=\frac{1}{\Gamma(n-\alpha)} \int_{t_{0}}^{t}(t-\xi)^{n-\alpha-1} \frac{\partial^{n} g(x, \xi)}{\partial \xi^{n}} d \xi, \quad t_{0} \geq 0, t>t_{0},
$$

where $n-1<\alpha \leq n, n \in \mathrm{N}$. In particularly, for $0<\alpha \leq 1$, Caputo partial fractional derivative becomes

$$
{ }^{C} D_{t}^{\alpha} g(x, t)=\frac{1}{\Gamma(1-\alpha)} \int_{t_{0}}^{t}(t-\xi)^{-\alpha} \frac{\partial g(x, \xi)}{\partial \xi} d \xi .
$$

Definition 3. The two-parameter Mittag-Leffler function defined as follow:

$$
E_{\alpha, \beta}(x)=\sum_{n=0}^{\infty} \frac{x^{n}}{\Gamma(n \alpha+\beta)}, \quad \alpha, \beta>0,
$$

if $\beta=1$, then this function is one-parameter Mittag-Leffler (i.e. $\left.E_{\alpha}(\cdot)\right)$ and if $\alpha=\beta=1$ this function 
represent the exponential function.

Lemma 1. The Caputo fractional derivative of generalized Mittag-Leffler function is given by

$$
{ }^{C} D_{t}^{\alpha} E_{\alpha}\left(A t^{\alpha}\right)={ }^{C} D_{t}^{\alpha}\left(\sum_{n=0}^{\infty} \frac{A^{n} t^{n \alpha}}{\Gamma(n \alpha+1)}\right)=\sum_{n=1}^{\infty} \frac{A^{n} t^{(n-1) \alpha}}{\Gamma((n-1) \alpha+1)}=\sum_{n=0}^{\infty} \frac{A^{n+1} t^{n \alpha}}{\Gamma(n \alpha+1)}=A E_{\alpha}\left(A t^{\alpha}\right),
$$

where $A$ is an undetermined coefficient.

Theorem 1. Ghorbani (2009) Assume that $N(v)$ is a nonlinear function, and $v=\sum_{l=0}^{\infty} \eta^{l} v_{l}$, then we have

$$
\frac{\partial^{n}}{\partial \eta^{n}} N(v)_{\eta=0}=\frac{\partial^{n}}{\partial \eta^{n}} N\left(\sum_{l=0}^{\infty} \eta^{l} v_{l}\right)_{\eta=0}=\frac{\partial^{n}}{\partial \eta^{n}} N\left(\sum_{l=0}^{n} \eta^{l} v_{l}\right)_{\eta=0}
$$

\section{Analysis of the MGMLFM}

This section explains the basic idea of MGMLFM, in order to do this, we consider a general nonlinear fractional partial system with initial conditions of the form

$$
{ }^{C} D_{t}^{\alpha} U(X, t)=L(U(X, t))+N(U(X, t),
$$

subject to the initial condition

$$
U(X, 0)=\phi(X)
$$

where ${ }^{C} D_{t}^{\alpha}$ is the arbitrary $(p-1<\alpha \leq p)$ order Caputo partial fractional derivative of function $U(X, t)$ with respect to " $t$ ", $U=\left(u_{1}, u_{2}, \cdots, u_{m}\right)^{T}$ whereas the superscript "T" means " transpose ", $X=\left(x_{1}, x_{2}, \cdots, x_{n}\right) \in \mathrm{R}^{n}, \quad p, m, n \in \mathrm{N}, L$ and $N$ are represented the general linear and nonlinear differential operator for the function $U(X, t)$, respectively, and $\phi=\left(\phi_{1}, \phi_{2}, \cdots, \phi_{m}\right)^{T}$ is a known function of variable $X$.

The MGMLFM proposed that the solution of $U(X, t)$ which announced in (4) can be written by an infinite series as the following

$$
\begin{aligned}
& u_{1}(X, t)=w_{1}(X) E_{\alpha}\left(A_{1} t^{\alpha}\right)=\sum_{j=0}^{\infty} w_{1}(X) A_{1}^{j} \frac{t^{j \alpha}}{\Gamma(j \alpha+1)}, \\
& u_{2}(X, t)=w_{2}(X) E_{\alpha}\left(A_{2} t^{\alpha}\right)=\sum_{j=0}^{\infty} w_{2}(X) A_{2}^{j} \frac{t^{j \alpha}}{\Gamma(j \alpha+1)}, \\
& \vdots \\
& u_{m}(X, t)=w_{m}(X) E_{\alpha}\left(A_{m} t^{\alpha}\right)=\sum_{j=0}^{\infty} w_{m}(X) A_{m}^{j} \frac{t^{j \alpha}}{\Gamma(j \alpha+1)},
\end{aligned}
$$

where $A_{1}, A_{2}, \cdots, A_{m}$ are undetermined coefficients and $w_{1}(X), w_{2}(X), \cdots, w_{m}(X)$ are a functions of the variable $X$. By using the initial condition (5) we have $w_{1}(X)=\phi_{1}(X), w_{2}(X)=\phi_{2}(X), \cdots, w_{m}(X)=\phi_{m}(X)$. Moreover, from Lemma 1, MGMLFM assumptions (6), the fractional partial system (4) and initial conditions (5) we have

$$
\sum_{j=0}^{\infty} \phi_{m}(X) A_{m}^{j+1} \frac{t^{j \alpha}}{\Gamma(j \alpha+1)}=L\left(\sum_{j=0}^{\infty} \phi_{m}(X) A_{m}^{j} \frac{t^{j \alpha}}{\Gamma(j \alpha+1)}\right)+N\left(\sum_{j=0}^{\infty} \phi_{m}(X) A_{m}^{j} \frac{t^{j \alpha}}{\Gamma(j \alpha+1)}\right), m=1,2, \cdots
$$

So, the linear term can be decomposed as

$$
L(U(X, t))=L\left(\sum_{j=0}^{\infty} \phi_{m}(X) A_{m}^{j} \frac{t^{j \alpha}}{\Gamma(j \alpha+1)}\right)=L\left(\phi_{m}(X)\right) \sum_{j=0}^{\infty} A_{m}^{j} \frac{t^{j \alpha}}{\Gamma(j \alpha+1)}
$$




$$
=\lambda_{m}^{*} \phi_{m}(X) \sum_{j=0}^{\infty} A_{m}^{j} \frac{t^{j \alpha}}{\Gamma(j \alpha+1)}, \quad m=1,2, \cdots,
$$

where $\lambda_{m}^{*}$ is a constant. With the help of Theorem 1 and He's polynomials Ghorbani (2009), Mohyud-Din et al. (2009), Liu (2012) fractional nonlinear equations by He polynomials the nonlinear term can be decomposed as

$$
N(U(X, t))=N\left(\sum_{j=0}^{\infty} \phi_{m}(X) A_{m}^{j} \frac{t^{j \alpha}}{\Gamma(j \alpha+1)}\right)=N\left(\phi_{m}(X)\right) N\left(\sum_{j=0}^{m} u_{j}(X, t)\right)
$$

By substituting from equations (8) and (9) into equation (7), we can identify the recurrence relation and obtain the undetermined coefficient $A_{m}$ and then get the general solution for the nonlinear fractional partial system.

\section{The Asymptotic Stability of the Equilibrium Points}

To determine the stability of the equilibrium points of a fractional order predator-prey model (1), we first evaluate the uniform steady states of the following system:

$$
\left\{\begin{array}{l}
{ }^{C} D_{t}^{\alpha} u_{1}(x, y, t)=f_{1}\left(u_{1}, u_{2}\right)+\frac{\partial^{2} u_{1}}{x^{2}}+\frac{\partial^{2} u_{1}}{y^{2}} \\
{ }^{C} D_{t}^{\alpha} u_{2}(x, y, t)=f_{2}\left(u_{1}, u_{2}\right)+\frac{\partial^{2} u_{2}}{x^{2}}+\frac{\partial^{2} u_{2}}{y^{2}},
\end{array}\right.
$$

where $(U, V)=\left(u_{1}, u_{2}\right), \quad f_{1}\left(u_{1}, u_{1}\right)=a u_{1}-b u_{1} u_{2} \quad$ and $f_{2}\left(u_{1}, u_{2}\right)=b u_{1} u_{2}-c u_{2}$. To evaluate the equilibrium points of $(10)$, let

$$
{ }^{C} D_{t}^{\alpha} u_{j}(x, y, t)=0, \quad j=1,2
$$

then $\left(u_{1}^{e q}, u_{2}^{e q}\right)=(0,0)$ (trivial) and $\left(u_{1}^{*}, u_{2}^{*}\right)=\left(\frac{c}{b}, \frac{a}{b}\right)$ (coexistence) are the equilibrium points, where $f_{j}\left(u_{1}^{e q}, u_{2}^{e q}\right)=f_{j}\left(u_{1}^{*}, u_{2}^{*}\right)=0, j=1,2$. For $\left(u_{1}^{*}, u_{2}^{*}\right)$, we examine the asymptotic stability Ahmed et al. (2004) by assume

$$
\left\{\begin{array}{l}
u_{j}=u_{j}^{*}+\grave{o}_{j} \exp (i k x+\lambda t), \\
u_{j}=u_{j}^{*}+\grave{o}_{j} \exp (i k y+\lambda t) .
\end{array} j=1,2\right.
$$

Thus, the eigenvalues are given by the following determinant

This can be solved for $\lambda$ as

$$
\left|\begin{array}{cc}
\frac{\partial f_{1}}{\partial u_{1}}-k^{2}-\lambda & \frac{\partial f_{1}}{\partial u_{2}} \\
\frac{\partial f_{2}}{\partial u_{1}} & \frac{\partial f_{2}}{\partial u_{2}}-k^{2}-\lambda
\end{array}\right|_{\left(u_{1}^{*}, u_{1}^{*}\right)}=0
$$

$$
\lambda_{1,2}(k)=\frac{t r_{k} \pm \sqrt{t r_{k}^{2}-4 \Delta_{k}}}{2}
$$


where, $t r_{k}$ refers to the trace of the following matrix

$$
M=\left(\begin{array}{cc}
\frac{\partial f_{1}}{\partial u_{1}}-k^{2} & \frac{\partial f_{1}}{\partial u_{2}} \\
\frac{\partial f_{2}}{\partial u_{1}} & \frac{\partial f_{2}}{\partial u_{2}}-k^{2}
\end{array}\right)_{\left(u_{1}^{*}, u_{2}^{*}\right)}
$$

and $\Delta_{k}$ to the determinate of $M$ (i.e. $\operatorname{tr}=\operatorname{tr}(M)$ and $\left.\Delta_{k}=\operatorname{det}(M)\right)$. The equilibrium point $\left(u_{1}^{*}, u_{2}^{*}\right)=\left(U^{*}, V^{*}\right)$ is locally asymptotically stable if both the eigenvalues $\lambda_{1}, \lambda_{2}$ are negative $\left(\left|\arg \left(\lambda_{i}\right)\right|>\frac{\alpha \pi}{2}, \forall i=1,2\right.$ (see e.g. Ahmed et al. (2006), Ameen (2018)).

\section{Applications and Results}

In this section, we apply the MGMLFM to solve the fractional predator-prey system in different cases and compare the results with other methods to illustrate the advantages and the accuracy of the MGMLFM.

Case 1. Here, we considered the system which announced in (1) with the following initial conditions

$$
U(x, y, 0)=U_{0}, \quad V(x, y, 0)=V_{0}
$$

Now we apply the MGMLFM. By using the suggestion stated in (6), let

$$
\begin{aligned}
& U(x, y, t)=\sum_{n=0}^{\infty} W_{1}(x, y) A^{n} \frac{t^{n \alpha}}{\Gamma(n \alpha+1)}, \\
& V(x, y, t)=\sum_{n=0}^{\infty} W_{2}(x, y) B^{n} \frac{t^{n \alpha}}{\Gamma(n \alpha+1)},
\end{aligned}
$$

where $A, B$ are undetermined coefficients. From the initial condition (12), we have $W_{1}(x, y)=U_{0}$ and $W_{2}(x, y)=V_{0}$. By using (8), we obtain the linear part of the system (1) as the following

$$
\begin{array}{ccc}
\Lambda\left(U_{0}\right)=a U_{0} & \Rightarrow & \lambda_{1}^{*}=a, \\
\Lambda\left(V_{0}\right)=-c V_{0} & \Rightarrow \quad \lambda_{2}^{*}=-c .
\end{array}
$$

Similarly, the nonlinear part of this system satisfies

$$
\Lambda\left(U_{0}\right)=-b U_{0} V_{0}, \quad \Lambda\left(V_{0}\right)=b U_{0} V_{0} .
$$

By using equations (7) and (1), then we have

$$
\begin{aligned}
& U_{0} \sum_{n=0}^{\infty}\left(A^{n+1}-a A^{n}+b V_{0} d^{n} \Gamma(n \alpha+1)\right) \frac{t^{n \alpha}}{\Gamma(n \alpha+1)}=0, \\
& V_{0} \sum_{n=0}^{\infty}\left(B^{n+1}+c B^{n}-b U_{0} d^{n} \Gamma(n \alpha+1)\right) \frac{t^{n \alpha}}{\Gamma(n \alpha+1)}=0,
\end{aligned}
$$

where 


$$
d^{n}=\sum_{k=0}^{n} \frac{A^{k} B^{n-k}}{\Gamma(k \alpha+1) \Gamma((n-k) \alpha+1)} .
$$

Then, the recurrence relations are defined by

$$
\begin{aligned}
& A^{n+1}=a A^{n}-b V_{0} d^{n} \Gamma(n \alpha+1), \\
& B^{n+1}=-c B^{n}+b U_{0} d^{n} \Gamma(n \alpha+1)
\end{aligned}
$$

By substitute different values of $n$, we obtain recursively

$$
\begin{array}{lc}
A^{0}=1, & B^{0}=1, \\
A^{1}=a-b V_{0}, & B^{1}=-c+b U_{0}, \\
A^{2}=a A^{1}-b V_{0}\left(A^{1}+B^{1}\right), & B^{2}=-c B^{1}+b U_{0}\left(A^{1}+B^{1}\right) \\
A^{3}=a A^{2}-b V_{0}\left(A^{2}+B^{2}+A^{1} B^{1} \frac{\Gamma(2 \alpha+1)}{(\Gamma(\alpha+1))^{2}}\right), & \\
B^{3}=-c B^{2}+b U_{0}\left(A^{2}+B^{2}+A^{1} B^{1} \frac{\Gamma(2 \alpha+1)}{(\Gamma(\alpha+1))^{2}}\right),
\end{array}
$$

From (13) and (14), we obtain approximate solution in a series form as the following

$$
\begin{aligned}
& U(x, y, t)=U_{0}\left(A^{0}+A^{1} \frac{t^{\alpha}}{\Gamma(\alpha+1)}+A^{2} \frac{t^{2 \alpha}}{\Gamma(2 \alpha+1)}+A^{3} \frac{t^{3 \alpha}}{\Gamma(3 \alpha+1)}+\cdots\right), \\
& V(x, y, t)=V_{0}\left(B^{0}+B^{1} \frac{t^{\alpha}}{\Gamma(\alpha+1)}+B^{2} \frac{t^{2 \alpha}}{\Gamma(2 \alpha+1)}+B^{3} \frac{t^{3 \alpha}}{\Gamma(3 \alpha+1)}+\cdots\right)
\end{aligned}
$$


Table 1. Comparison of the numerical values with MGMLFM, HPM and VIM when $U_{0}=100, V_{0}=10$, $a=0.05, b=0.03$, and $c=0.01$

\begin{tabular}{llrrr}
\hline$t$ & $\alpha$ & Value $(U, V)$ by MGMLFM & Value $(U, V)$ by HPM & Value $(U, V)$ by VIM \\
\hline 0.02 & 1 & $(99.4831,10.6146)$ & $(99.4831,10.6146)$ & $(99.4834,10.6323)$ \\
& 0.9 & $(99.1865,10.9633)$ & $(99.1865,10.9633)$ & $(99.3065,10.8375)$ \\
0.2 & 1 & $(93.0910,17.8514)$ & $(93.0910,17.8514)$ & $(93.3908,17.7382)$ \\
& 0.9 & $(90.5735,20.5567)$ & $(90.5735,20.5567)$ & $(92.4584,18.8198)$ \\
& 1 & $(87.9348,23.4430)$ & $(87.9348,23.4430)$ & $(88.9466,22.7237)$ \\
& 0.9 & $(83.7933,27.7785)$ & $(83.7933,27.7785)$ & $(87.8005,24.0532)$ \\
\hline
\end{tabular}

This table shows the approximate solutions of the system (1) with initial conditions (12) by using MGMLFM, HPM and VIM when the parameter takes values $U_{0}=100, V_{0}=10, a=0.05, b=0.03$, and $c=0.01$.

Case 2. In this case, the initial conditions of the system (1) are given by

$$
U(x, y, 0)=e^{x+y}, \quad V(x, y, 0)=e^{x+y}
$$

Using the same suggestion in Case 1 for the functions $U(x, y, t)$ and $V(x, y, t)$ stated in (13) and (14), respectively. The linear part is given by

$$
\begin{aligned}
& \Lambda\left(e^{x+y}\right)=(2+a) e^{x+y} \Rightarrow \lambda_{1}^{*}=(2+a), \\
& \Lambda\left(e^{x+y}\right)=(2-c) e^{x+y} \Rightarrow \lambda_{2}^{*}=(2-c) \text {, }
\end{aligned}
$$

and nonlinear part of this case gives

$$
\Lambda\left(e^{x+y}\right)=-b e^{2 x+2 y}, \quad \Lambda\left(e^{x+y}\right)=b e^{2 x+2 y} .
$$

By using equation (7) we get

$$
\begin{aligned}
& e^{x+y} \sum_{n=0}^{\infty}\left(A^{n+1}-(2+a) A^{n}+b e^{x+y} d^{n} \Gamma(n \alpha+1)\right) \frac{t^{n \alpha}}{\Gamma(n \alpha+1)}=0, \\
& e^{x+y} \sum_{n=0}^{\infty}\left(B^{n+1}-(2-c) B^{n}-b e^{x+y} d^{n} \Gamma(n \alpha+1)\right) \frac{t^{n \alpha}}{\Gamma(n \alpha+1)}=0,
\end{aligned}
$$

where $d^{n}$ is defined before. Then, the recurrence relations are defined by

$$
\begin{aligned}
& A^{n+1}=(2+a) A^{n}-b e^{x+y} d^{n} \Gamma(n \alpha+1), \\
& B^{n+1}=(2-c) B^{n}+b e^{x+y} d^{n} \Gamma(n \alpha+1) .
\end{aligned}
$$

By substitute different values of $n$, we obtain recursively

$$
\begin{array}{ll}
A^{0}=1, & B^{0}=1, \\
A^{1}=2+a-b e^{x+y}, & B^{1}=2-c+b e^{x+y},
\end{array}
$$




$$
\begin{aligned}
& A^{2}=(2+a) A^{1}-b e^{x+y}\left(A^{1}+B^{1}\right), \quad B^{2}=(2-c) B^{1}+b e^{x+y}\left(A^{1}+B^{1}\right), \\
& A^{3}=(2+a) A^{2}-b e^{x+y}\left(A^{2}+B^{2}+A^{1} B^{1} \frac{\Gamma(2 \alpha+1)}{(\Gamma(\alpha+1))^{2}}\right), \\
& B^{3}=(2-c) B^{2}+b e^{x+y}\left(A^{2}+B^{2}+A^{1} B^{1} \frac{\Gamma(2 \alpha+1)}{(\Gamma(\alpha+1))^{2}}\right) .
\end{aligned}
$$

The approximation solution in a series form is given by

$$
\begin{aligned}
& U(x, y, t)=e^{x+y}\left(A^{0}+A^{1} \frac{t^{\alpha}}{\Gamma(\alpha+1)}+A^{2} \frac{t^{2 \alpha}}{\Gamma(2 \alpha+1)}+A^{3} \frac{t^{3 \alpha}}{\Gamma(3 \alpha+1)}+\cdots\right), \\
& V(x, y, t)=e^{x+y}\left(B^{0}+B^{1} \frac{t^{\alpha}}{\Gamma(\alpha+1)}+B^{2} \frac{t^{2 \alpha}}{\Gamma(2 \alpha+1)}+B^{3} \frac{t^{3 \alpha}}{\Gamma(3 \alpha+1)}+\cdots\right) .
\end{aligned}
$$
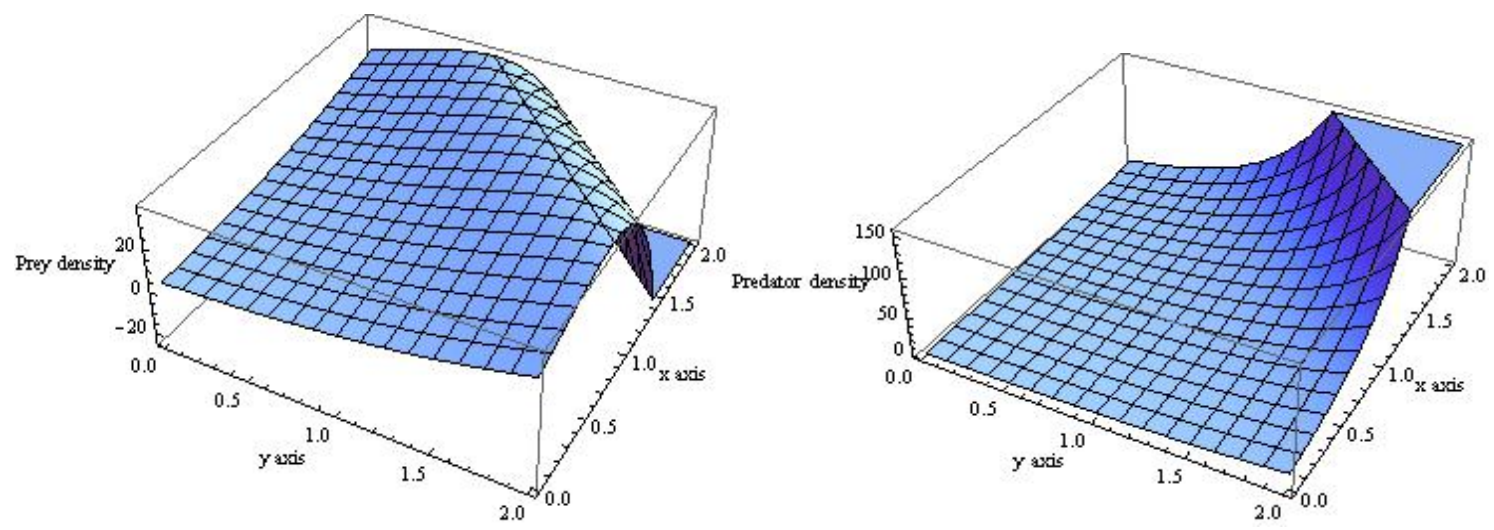

Figure 1. The surface displays the solution of $U(x, y, t)$ and $V(x, y, t)$ by using MGMLFM for $\alpha=1$, $a=0.7, b=0.03, c=0.3$ and $t=0.53$.

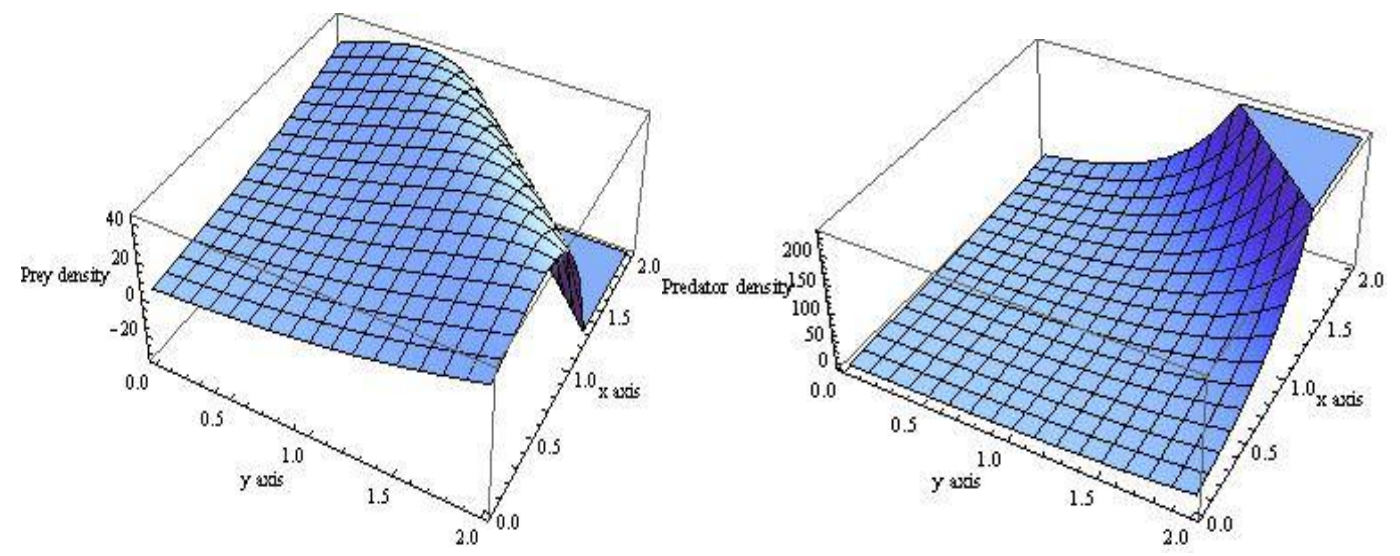

Figure 2. The surface displays the solution of $U(x, y, t)$ and $V(x, y, t)$ by using "MGMLFM" for

$$
\alpha=0.85, \quad a=0.7, \quad b=0.03, \quad c=0.3 \text { and } t=0.53 \text {. }
$$



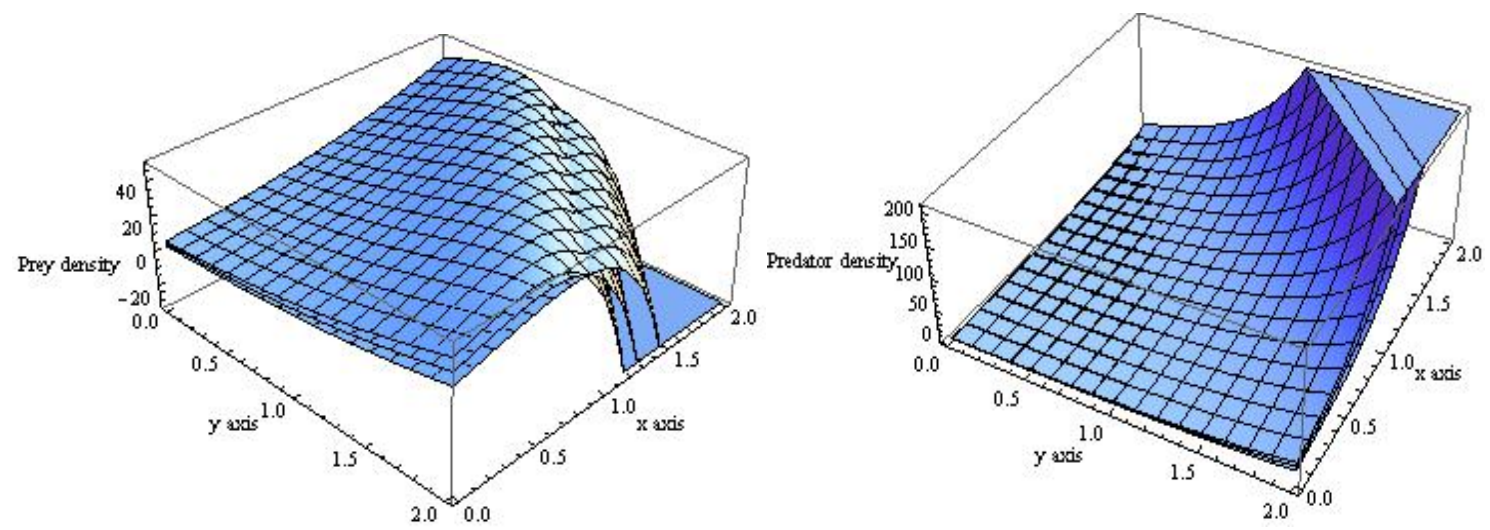

Figure 3. The surface displays the solution of $U(x, y, t)$ and $V(x, y, t)$ by using MGMLFM for $\alpha=1,0.85,0.75 \quad a=0.7, \quad b=0.03, \quad c=0.3$ and $t=0.53$.
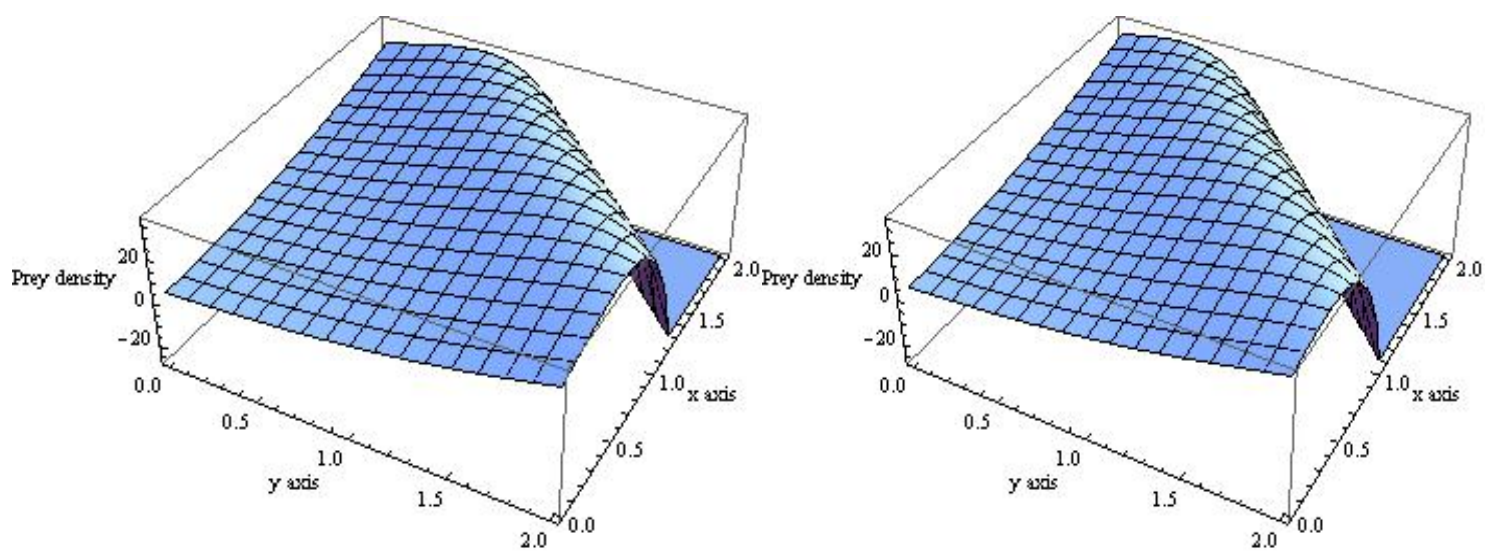

Figure 4. The surface displays the solution of $U(x, y, t)$ by using MGMLFM for $\alpha=0.85, c=0.3$, $t=0.53, a=0.5, b=0.03$ in the drawing on the left and $a=0.7, b=0.04$ in the drawing on the right.

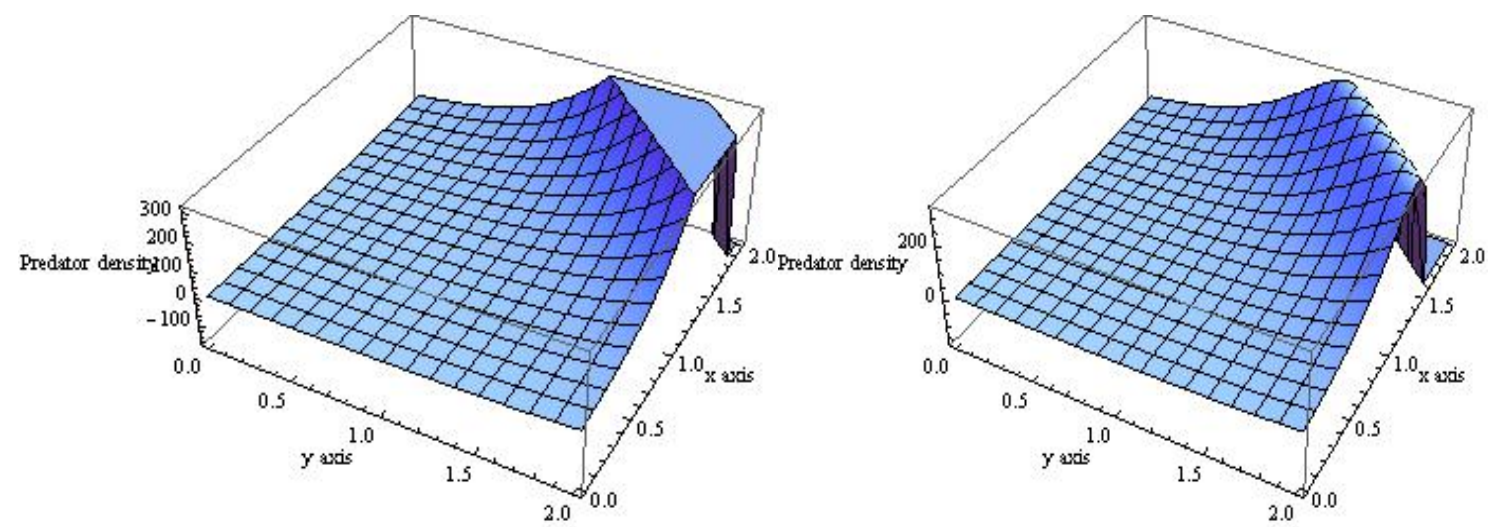

Figure 5. The surface displays the solution of $V(x, y, t)$ by using "MGMLFM" for $\alpha=0.85, a=0.5$, $t=0.53, c=0.6, b=0.04$ in the drawing on the left and $c=0.8, b=0.06$ in the drawing on the right.

We presented in Figure 1 the numerical simulation of the proposed system with a fitting parameter. From the graphical results, we realize that $U(x, y, t)$ first increases with the spatial factors (e.g. In some places, prey 
are vulnerable to predation while in the other places is a complete shelter, see for more details Křivan (2008)), after that decreases. In spite of the fact that $V(x, y, t)$ always increases with the spatial factors with the selected parameters. Figure 3 shows the numerical simulation of the prey-predator population density with different values of $\alpha$. Figures 4 and 5 reflect the effect of various estimations of parameters $a, b$ and $c$ on $U(x ; y ; t)$ and $V(x ; y ; t)$. Comparing with the previous figures, we have reached the parameter $a, b$ infects the increased speed, the maximum esteem, and the decrease speed of $U(x, y, t)$. While, the parameters $c$ and $b$ impact on $V(x, y, t)$ where the climax takes a small time to be reduced. This behavior corresponds to the results in our real world. From the above results of the fractional prey-predator population system, show that the fractional model coincides with the anomalous biological diffusion behavior spotted in the domain.

\section{Conclusion}

This article deals with partial differential equations that contain time-fractional derivatives for predator-prey modeling which describes a biological population dynamics system and its approximate solution is presented using relatively new analytical techniques MGMLFM. The numerical results for the fractional order predator-prey model with different values of parameter $a, b$ and $c$ are agreed with the theoretical analysis of the equilibrium points and their local asymptotic stability. In order to assess the advantages and the accuracy of the MGMLFM we compare our results with the results have been done previously by some other well-known methods. From the results, we have seen that this methodology is an efficient and proficient method for finding the analytical and approximate solution for nonlinear partial differential equations of fractional order.

\section{References}

Adomian, G. (1988). A review of the decomposition method in applied mathematics. J. Math. Anal. Appl., 135, 501-544. https://doi.org/10.1016/0022-247X(88)90170-9.

Ahmed, E., Hegazi, A. \& Elgazzar, A. (2004). On persistence and stability of some biological systems with cross diffusion. Advances in Complex Systems, 7, 65-76. https://doi.org/10.1142/S0219525904000056.

Ahmed, E., El-Sayed, A.M.A. \& El-Saka, H.A.A. (2006). On some Routh-Hurwitz conditions for fractional order differential equations and their applications in Lorenz, Rössler, Chua and Chen systems. Phys. Lett. A, 358, 1-4. https://doi.org/10.1016/j.physleta.2006.04.087.

Ahmed, E., El-Sayed, A. M. A. \& El-Saka, H. A. A. (2007). Equilibrium points, stability and numerical solutions of fractional-order predator-prey and rabies models. J. Math. Anal. Appl., 325, 542-553. https://doi.org/10.1016/j.jmaa.2006.01.087.

Arafa, A. A. M., Rida, S. Z. \& Ali, H. M. (2013a). Generalized Mittag-Leffler function method for solving Lorenz system. International Journal of Innovation and Applied Studies, 3, 105-111. http://www.issr-journals.org/ijias/.

Arafa, A. A. M., Rida, S. Z., Mohammadein, A. A. \& Ali, H. M. (2013). solving nonlinear fractional differential equation by generalized Mittag-Leffler function method. Communications in Theoretical Physics, 59, 661-663. https://doi.org/10.1088/0253-6102/59/6/01

Ameen, I. (2018). The effect of vaccination and treatment of measles disease described by a fractional order model. World Journal of Modelling and Simulation, 14, 30-38.

Du, M., Wang, Z. \& Hu H. (2013). Measuring memory with the order of fractional derivative. Sci. Rep., 3, 1-3. https://doi.org/10.1038/srep03431

El-Saka, H. A. A. (2014). Fractional-order partial differential equation for perdator-pery. Journal of Fractional Calculus and Applications, 5, 44-51. http://fcag-egypt.com/Journals/JFCA/.

Ganji, D. D. \& Sadighi, A. (2007). Application of homotopy perturbation and variational iteration methods to nonlinear heat transfer and porous media equations. J. Comput. Appl. Math., 207, 699-708. https://doi.org/10.1016/j.cam.2006.07.030.

Ghorbani, A. (2009). Beyond Adomian polynomials: He polynomials. Chaos, Solitons and Fractals, 39(3), 1486-1492. https://doi.org/10.1016/j.chaos.2007.06.034.

Gourley, S. A. \& Britton, N. F. (1996). A predator-prey reaction-diffusion system with nonlocal effects. Journal of Mathematical Biology, 34, 297-333. https://doi.org/10.1007/BF00160498.

He, J. H. (2005). Application of homotopy perturbation method to nonlinear wave equation. Chaos Solitons 
Fractals, 26, 695-700. https://doi.org/10.1016/j.chaos.2005.03.006.

He, J. H. (2011). A short remark on fractional variational iteration method. Phys. Lett. A, 375, 3362-3364. https://doi.org/10.1016/j.physleta.2011.07.033.

Hilfer, R. (2000). Applications of Fractional Calculus in Physics. World Scientific, Singapore. https://oi.org/10.1142/9789812817747

Kadem, A. \& Baleanu, D. (2011). Homotopy perturbationmethod for the coupled fractional Lotka-Volterra equations. Romanian Journal of Physics, 56, 332-338.

Křivan, V. (2008). Prey-Predator Models. Encyclopedia of Ecology, 2929-2940. https://doi.org/10.1016/B978-008045405-4.00676-5

Liu, Y. \& Xin, B. (2011). Numerical solutions of a fractional predator-prey system. Advances in Difference Equations, 2011, 1-11. https://doi.org/10.1155/2011/190475.

Liu, Y. Q. (2012). Approximate solutions of fractional nonlinear equations using homotopy perturbation transformation method. Abstract and Applied Analysis, 2012, Article ID 752869, 14 pages. https://doi.org/10.1155/2012/752869

Liu, Y., Sun, H., Yin, X. \& Xin, B. (2017). A new Mittag-Leffler function undetermined coefficient method and its applications to fractional homogeneous partial differential equations. J. Nonlinear Sci. Appl., 10, 4515-4523. https://doi.org/10.22436/jnsa.010.08.43

Metzler, R. \& Klafter, J. (2000). The random walk's guide to anomalous diffusion: a fractional dynamics approach. Physics Reports A, 339, 1-77. https://doi.org/10.1016/S0370-1573(00)00070-3.

Moaddy, K., Radwan, A.G., Salama, K.N. \& Momani, S. (2012). The fractional-order modeling and synchronization of electrically coupled neuron systems. Comput. Math. Appl., 64, 3329-3339. https://doi.org/10.1016/j.camwa.2012.01.005.

Mohyud-Din, S. T., Noor, M. A. \& Noor, K. I. (2009). Traveling wave solutions of seventh-order generalized $\mathrm{KdV}$ equations using He's polynomials. International Journal of Nonlinear Sciences and Numerical Simulation, 10(2), 227-233. https://doi.org/10.1515/IJNSNS.2009.10.2.227.

Oldham, K. B. \& Spanier, J. (1974). The Fractional Calculus. Academic Press, New York.

Petras, I. (2011). Fractional-Order Nonlinear Systems, Modeling, Analysis and Simulation, Springer. Nonlinear Physical Science ISBN 978-7-04-031534-9.

Petrovskii, S., Malchow, H. \& Li, B. (2005). An exact solution of a diffusive predator-prey system. Proceedings of The Royal Society of London A, 461, 1029-1053. https://doi.org/10.1098/rspa.2004.1404.

Podlubny, I. (1999). Fractional Differential Equations, Mathematics in Sciences and Engineering, 198, Academic Press, San Diego.

Safari, M., Ganji, D. D. \& Moslemi, M. (2009). Application of He's variational iteration method and Adomian's decomposition method to the fractional KdV-Burgers-Kuramoto equation. Comput. Math. Appl., 58, 2091-2097. https://doi.org/10.1016/j.camwa.2009.03.043.

Samko, S. G., Kilbas, A. A. \& Marichev, O. I. (1993). Fractional Integrals and Derivatives: Theory and Applications. Switzerland ; Philadelphia, Pa., USA : Gordon and Breach Science Publishers.

\section{Copyrights}

Copyright for this article is retained by the author(s), with first publication rights granted to the journal.

This is an open-access article distributed under the terms and conditions of the Creative Commons Attribution license (http://creativecommons.org/licenses/by/3.0/). 